\title{
On complete decompositions of dihedral groups
}

\author{
Huey Voon Chen $^{1 *}$, and Chang Seng $\operatorname{Sin}^{1}$ \\ ${ }^{1}$ Department of Mathematical and Actuarial Sciences, Lee Kong Chian Faculty of Engineering and \\ Science, Universiti Tunku Abdul Rahman, 43000 Kajang, Selangor, Malaysia
}

\begin{abstract}
Let $G$ be a finite non-abelian group and $B_{1}, \ldots, B_{t}$ be nonempty subsets of $G$ for integer $t \geq 2$. Suppose that $B_{1}, \ldots, B_{t}$ are pairwise disjoint, then $\left(B_{1}, \ldots, B_{t}\right)$ is called a complete decomposition of $G$ of order $t$ if the subset product $B_{i 1} \ldots B_{i t}=\left\{b_{i 1} \ldots b_{i t} \mid b_{i j} \in B_{i j}, j=1,2, \ldots, t\right\}$ coincides with $G$, where $\left\{B_{i 1}, \ldots, B_{i t}\right\}=\left\{B_{1}, \ldots, B_{t}\right\}$ and the $B_{i j}$ are all distinct. Let $D_{2 n}=\left\langle r, s \mid r^{n}=s^{2}=1, r s=s r^{n-1}\right\rangle$ be the dihedral group of order $2 n$ for integer $n \geq 3$. In this paper, we shall give the constructions of the complete decompositions of $D_{2 n}$ of order $t$, where $2 \leq t \leq n$.
\end{abstract}

\section{Introduction}

Many researchers have conducted their research related to group coverings over the years. There are many types of group coverings and group factorization is one of the group coverings. The first result that related to group factorization was showed in [1] and rediscovered by Davenport in 1935, see [2]. Hajos (see [3] and [4]) proposed a new method called group theoretical equivalent as a solution for Minkowski's geometry problem [5]. He reformulated it into a problem in group covering, and investigated how to factor a finite abelian group into certain subsets. As a result, people started to realize the importance of factoring group into subsets.

Over the years, there are numerous results related to group factorization. Brujin [6] conjectured that if $G=A+B$ is a factorization in which one of the factors has prime order and if $G$ is a finite cyclic group, then either $A$ or $B$ is periodic. Redei [7] showed that if a finite abelian group $G$ contains identity element, given each subsets of $G$ has a prime number of elements and $G$ is a direct product of its subsets, then he found that at least one of the subsets must be a subgroup.

We notice that the exhaustion numbers of subsets of dihedral groups, $D_{2 n}$, had been thoroughly studied, see ([8] and [9]). In this paper, we shall focus on investigating the complete decompositions of $D_{2 n}$. In year 2018, the existence of complete decompositions of cyclic group $\mathbb{Z}_{n}$ is determined, refer to [10]. By using the complete decompositions of cyclic groups, they found an application in constructing codes over a binary alphabet.

Throughout this paper, we let $G$ be dihedral groups, $D_{2 n}$, for integer $n \geq 3$. It is clear that $D_{2 n}=\left\langle r, s \mid r^{n}=s^{2}=1, s r=r^{-1} s\right\rangle=\left\{1, r, \ldots, r^{n-1}, s, r s, \ldots, r^{n-1} s\right\}$ is a finite nonabelian group of order $2 n$, where $\langle r\rangle=\left\{1, r, \ldots, r^{n-1}\right\}$ is a cyclic subgroup of $D_{2 n}$ of order $n$. Recall that the definition of complete decompositions of $G$ of order $t$ is as follows: Let $G$

*Corresponding author: chenhv@utar.edu.my 
be a finite non-abelian group and let $B_{1}, \ldots, B_{t}$ be nonempty subsets of $G$ for integer $t \geq 2$. Suppose that $B_{1}, \ldots, B_{t}$ are pairwise disjoint, then $\left(B_{1}, \ldots, B_{t}\right)$ is called a complete decomposition of $G$ of order $t$ if the subset product $B_{i 1} \ldots B_{i t}=\left\{b_{i 1} \ldots b_{i t} \mid b_{i j} \in B_{i j}, j=1,2, \ldots\right.$, $t\}$ coincides with $G$, where $\left\{B_{i 1}, \ldots, B_{i t}\right\}=\left\{B_{1}, \ldots, B_{t}\right\}$ and the $B_{i j}$ are all distinct.

Let $A, B \subseteq D_{2 n}$. If $A \cap B=\varnothing$ and either $|A|=1$ or $|B|=1$, then $|A B|<2 n$ and hence $(A, B)$ is not a complete decomposition of $D_{2 n}$ of order 2 . Thus, we consider the subsets $A, B$ such that $|A|,|B| \in\{2,3, \ldots, 2 n-2\}$. In this paper, we first provide some constructions of complete decompositions of $D_{2 n}$ of order 2 . We extend the results by showing some constructions of complete decompositions of $D_{2 n}$ of order $t$ for $t \in\{3,4, \ldots, n\}$.

\section{Constructions of complete decompositions of $D_{2 n}$ of order 2}

We begin by providing an example of complete decompositions of $D_{8}$ of order 2 . Let $A=$ $\left\{1, r, r^{2}, r^{3} s\right\}$ and $B=\left\{s, r s, r^{2} s, r^{3}\right\}$, where $A, B \subseteq D_{8}$. Then we see that $(A, B)$ is a complete decomposition of $D_{8}$ of order 2 , since $A B=D_{8}$.

By using the group relation in $D_{2 n}$, the result below is obvious.

Proposition 1. Let $i, n$ be integers, where $n \geq 3$ and $i \in\{0,1, \ldots, n-1\}$. If $r^{i}, s \in D_{2 n}$ then $s r^{i}=r^{n-i} s$.

\section{Proof.}

Since $s r=r^{-1} s$, it follows that $s r^{2}=s r r=r^{-1} s r=r^{-1} r^{-1} s=r^{-2} s$. Thus, by using induction on power $i$, we obtain $s r^{i}=r^{-i} s$. Since $r^{n}=1$, it follows that $r^{n-i}=r^{-i}$. Thus, we have $s r^{i}=r^{n-i} s$.

Proposition 2. Let $n \geq 3$ and $A=\left\{1, r, \ldots, r^{n-2}, s\right\}$ and $B=\left\{r^{n-1}, r s, r^{2} s, \ldots, r^{n-1} s\right\}$, where $A, B \subseteq D_{2 n}$. Then $(A, B)$ is a complete decomposition of $D_{2 n}$ of order 2 .

\section{Proof.}

Note that $A B=\left\{1, r, \ldots, r^{n-2}, s\right\}\left\{r^{n-1}, r s, \ldots, r^{n-1} s\right\}=D_{2 n}$. Hence, $(A, B)$ is a complete decomposition of $D_{2 n}$ of order 2 .

In the following, we show that there exists a complete decomposition of $D_{2 n}$ of order 2, where $A \cap B=\emptyset,|A| \neq|B|$ and $A \cup B=D_{2 n}$.

Proposition 3. Let $A=\left\{1, r^{v+1}, r^{v+2}, \ldots, r^{n-1}, r^{v} s, r^{v+1} s, \ldots, r^{n-1} s\right\}$ and $B=\left\{r, r^{2}, \ldots, r^{v}\right.$, $\left.s, r s, \ldots, r^{v-1} s\right\}$ be the subsets of $D_{2 n}$, where $v \in\{1,2, \ldots, n-2\},|A|=2 n-2 v$ and $|B|=$ $2 v$. Then $(A, B)$ is a complete decomposition of $D_{2 n}$ of order 2 for integer $n \geq 3$.

\section{Proof.}

Let $L_{1}=\left\{r^{v+1}, r^{v+2}, \ldots, r^{n-1}\right\} \subseteq A$ and $L_{2}=\left\{r, r^{2}, \ldots, r^{v}\right\} \subseteq B$. Note that $L_{1} L_{2}=$ $\left\{r^{v+1}, r^{v+2}, \ldots, r^{n-1}\right\}\left\{r, r^{2}, \ldots, r^{v}\right\}=\left\{r^{v+2}, r^{v+3}, \ldots, r^{n-1}\right\} \cup\left\{1, r, \ldots, r^{v-1}\right\}$.

Note that $1 \in A$, therefore $\left\{r, r^{2}, \ldots, r^{v}\right\} \subseteq A B$. It is clear that $\langle r\rangle \backslash\left(L_{1} L_{2} \cup\left\{r^{v}\right\}\right)=\left\{r^{v+1}\right\}$. Observe that $r^{v+1} s \in A$ and $s \in B$. Thus, we have $\left(r^{v+1} s\right)(s)=r^{v+1} \in A B$. Therefore, $\left\{r^{v}, r^{v+1}, \ldots, r^{v+n-1}\right\}=\langle r\rangle \subseteq A B$.

It remains to show that $\langle r\rangle_{s} \subseteq A B$. Let $L_{3}=\left\{s, r s, \ldots, r^{v-1} s\right\} \subseteq B$. We see that

$$
\begin{aligned}
L_{1} L_{3} & =\left\{r^{v+1}, r^{v+2}, \ldots, r^{n-1}\right\}\left\{s, r s, \ldots, r^{v-1} s\right\} \\
& =\left\{r^{v+1} s, r^{v+2} s, \ldots, r^{n-1} s\right\} \cup\left\{s, r s, \ldots, r^{v-2} s\right\} .
\end{aligned}
$$

Clearly $\langle r\rangle s \backslash L_{1} L_{3}=\left\{r^{v} s, r^{v+n-1} s\right\}$. Observe that $\left\{r^{v} s, r^{v+1} s\right\} \subseteq A$ and $r \in B$. Therefore, we have $\left\{r^{v} s, r^{v+1} s\right\} r=\left\{r^{v+n-1} s, r^{v+n} s\right\}=\left\{r^{v} s, r^{v-1} s\right\} \subseteq A B$. Thus, $\left\{r^{v-1} s, r^{v} s, \ldots\right.$, 
$\left.r^{v+n-2} s\right\}=\langle r\rangle_{S} \subseteq A B$. Hence, we conclude that $(A, B)$ is a complete decomposition of $D_{2 n}$ of order 2 .

Next, a construction of complete decompositions of $D_{2 n}$ of order 2, where $A \cap B=$ $\emptyset,|A| \neq|B|$ and $A \cup B \subset D_{2 n}$ is shown below.

Proposition 4. Let $n \geq 6$ be an even integer. Let $A=\left\{1, r, \ldots, r^{n-3}, r^{n-2} s, r^{n-1} s\right\}$ and $B_{i}=$ $\left\{r^{n-2}, r^{n-1}, s, r s, \ldots, r^{n-3} s\right\} \backslash\left\{r s, r^{3} s, \ldots, r^{i} s\right\}$, where $A, B_{j} \subseteq D_{2 n}, j \in\{1,3, \ldots, n-5\}$, $|A|=n$ and $|B|=n-\frac{j+1}{2}$. Then $\left(A, B_{j}\right)$ is a complete decomposition of $D_{2 n}$ of order 2 .

\section{Proof.}

Note that

$$
\begin{aligned}
B_{n-5} & =\left\{r^{n-2}, r^{n-1}, s, r^{2} s, \ldots, r^{n-4} s, r^{n-3} s\right\}, \\
B_{n-7} & =\left\{r^{n-2}, r^{n-1}, s, r^{2} s, \ldots, r^{n-6} s, r^{n-5} s, r^{n-4} s, r^{n-3} s\right\}, \\
& \vdots \\
B_{1} & =\left\{r^{n-2}, r^{n-1}, s, r^{2} s, r^{3} s, \ldots, r^{n-3} s\right\},
\end{aligned}
$$

where $B_{n-5} \subseteq B_{n-7} \subseteq \cdots \subseteq B_{1}$. Therefore, we shall focus on proving that $A B_{n-5}=D_{2 n}$. Let $L_{1}=\left\{1, r, \ldots, r^{n-3}\right\} \subseteq A$ and $L_{2}=\left\{s, r^{2} s\right\} \subseteq B_{n-5}$. We compute $L_{1} L_{2}$ as follows:

$$
\begin{aligned}
L_{1} L_{2} & =\left\{1, r, \ldots, r^{n-3}\right\}\left\{s, r^{2} s\right\} \\
& =\left\{s, r s, \ldots, r^{n-3} s, r^{2} s, r^{3} s, \ldots, r^{n-1} s\right\}=\langle r\rangle s .
\end{aligned}
$$

Therefore $\langle r\rangle s \subseteq A B_{n-5}$. Now, we let $L_{3}=\left\{r^{n-2}, r^{n-1}\right\} \subseteq B_{n-5}$. Then, we compute $L_{1} L_{3}$ as follows:

$$
L_{1} L_{3}=\left\{1, r, \ldots, r^{n-3}\right\}\left\{r^{n-2}, r^{n-1}\right\}=\left\{1, r, \ldots, r^{n-4}, r^{n-2}, r^{n-1}\right\} .
$$

Note that the product of $r^{n-1} s \in A$ and $r^{2} s \in B_{n-5}$ gives us $\left(r^{n-1} s\right)\left(r^{2} s\right)=r^{n-3} \in A B_{n-5}$. Therefore, $\langle r\rangle \subseteq A B_{n-5}$. Since $A B_{n-5}=D_{2 n}$, we see that $\left(A, B_{n-5}\right)$ is a complete decomposition of $D_{2 n}$ of order 2. Since $A B_{n-5}=D_{2 n}$ and $B_{n-5} \subseteq B_{n-7} \subseteq \cdots \subseteq B_{1}$, it follows that $A B_{n-5}=A B_{n-7}=\cdots=A B_{1}=D_{2 n}$. Hence, we conclude that $\left(A, B_{j}\right)$ is a complete decomposition of $D_{2 n}$ of order 2 for $j \in\{1,3, \ldots, n-5\}$.

\section{Constructions of complete decompositions of $D_{2 n}$ of order $k$ for $k \in\{3,4, \ldots, n\}$}

By using Matlab, we first generate all the possible complete decomposition of $D_{8}$ of order 3 . We found that there are total of 368 constructions show that $\left(B_{1}, B_{2}, B_{3}\right)$ is a complete decomposition of $D_{8}$ of order 3. Since dihedral group is a non-abelian group, the product of the subsets might not be the same. Hence, we check the product of the subsets $B_{1} B_{2} B_{3}$ and $B_{2} B_{1} B_{3}$. We notice that there are 17 out of 368 constructions indicate that $B_{1} B_{2} B_{3} \neq B_{2} B_{1} B_{3}$. To be more precise, Table 1 showed that there are 17 constructions where $\left(B_{1}, B_{2}, B_{3}\right)$ is a complete decomposition of $D_{8}$ of order 3 but $\left(B_{2}, B_{1}, B_{3}\right)$ is not a complete decomposition of $D_{8}$ of order 3 . 
Table 1. List of complete decompositions of $D_{8}$ of order 3 , where $B_{1}, B_{2}, B_{3} \subseteq D_{8}$ and $B_{1} B_{2} B_{3} \neq B_{2} B_{1} B_{3}$.

\begin{tabular}{|c|c|c|}
\hline$B_{1}$ & $B_{2}$ & $B_{3}$ \\
\hline$\{1, s\}$ & $\{r, r s\}$ & $\left\{r^{2}, r^{3}, r^{2} s, r^{3} s\right\}$ \\
\hline$\{1, s\}$ & $\left\{r^{3}, r^{3} s\right\}$ & $\left\{r, r^{2}, r s, r^{2} s\right\}$ \\
\hline$\{1, r s\}$ & $\left\{r, r^{2} s\right\}$ & $\left\{r^{2}, r^{3}, s, r^{3} s\right\}$ \\
\hline$\{1, r s\}$ & $\left\{r^{3}, s\right\}$ & $\left\{r, r^{2}, r^{2} s, r^{3} s\right\}$ \\
\hline$\left\{1, r^{2} s\right\}$ & $\left\{r, r^{3} s\right\}$ & $\left\{r^{2}, r^{3}, s, r s\right\}$ \\
\hline$\left\{1, r^{2} s\right\}$ & $\left\{r^{3}, r s\right\}$ & $\left\{r, r^{2}, s, r^{3} s\right\}$ \\
\hline$\left\{1, r^{3} s\right\}$ & $\{r, s\}$ & $\left\{r^{2}, r^{3}, r s, r^{2} s\right\}$ \\
\hline$\left\{1, r^{3} s\right\}$ & $\left\{r^{3}, r^{2} s\right\}$ & $\left\{r, r^{2}, s, r s\right\}$ \\
\hline$\left\{r, r^{2}\right\}$ & $\{1, s\}$ & $\left\{r^{3}, r s, r^{2} s, r^{3} s\right\}$ \\
\hline$\left\{r^{2}, r s\right\}$ & $\{r, s\}$ & $\left\{1, r^{3}, r^{2} s, r^{3} s\right\}$ \\
\hline$\left\{r^{2}, r^{2} s\right\}$ & $\{r, r s\}$ & $\left\{1, r^{3}, s, r^{3} s\right\}$ \\
\hline$\left\{r^{2}, r^{3} s\right\}$ & $\left\{r, r^{2} s\right\}$ & $\left\{1, r^{3}, s, r s\right\}$ \\
\hline$\left\{r^{2}, s\right\}$ & $\left\{r, r^{3} s\right\}$ & $\left\{1, r^{3}, r s, r^{2} s\right\}$ \\
\hline$\left\{r^{2}, s\right\}$ & $\left\{r^{3}, r s\right\}$ & $\left\{1, r, r^{2} s, r^{3} s\right\}$ \\
\hline$\left\{r^{2}, r s\right\}$ & $\left\{r^{3}, r^{2} s\right\}$ & $\left\{1, r, s, r^{3} s\right\}$ \\
\hline$\left\{r^{2}, r^{2} s\right\}$ & $\left\{r^{3}, r^{3} s\right\}$ & $\{1, r, s, r s\}$ \\
\hline$\left\{r^{2}, r^{3} s\right\}$ & $\left\{r^{3}, s\right\}$ & $\left\{1, r, r s, r^{2} s\right\}$ \\
\hline
\end{tabular}

Therefore, we remark that if $\left(B_{1}, B_{2}, \ldots, B_{t}\right)$ is a complete decomposition of $D_{2 n}$ of order $t$, it does not imply that $\left(B_{j_{1}}, B_{j_{2}}, \ldots, B_{j_{t}}\right)$ is a complete decomposition of $D_{2 n}$ of order $t$, where $\left\{B_{j_{1}}, B_{j_{2}}, \ldots, B_{j t}\right\}=\left\{B_{1}, B_{2}, \ldots, B_{t}\right\}$.

Now, we provide a construction of complete decompositions of $D_{2 n}$ of order 3 as follow.

Proposition 5. Let $n \geq 3$ and $A_{1}, A_{2}, A_{3} \subseteq D_{2 n}$, where $A_{1}=\{1, r\}, A_{2}=\{s, r s\}$ and $A_{3}=$ $\left\{r^{2}, r^{3}, \ldots, r^{n-1}, r^{2} s, r^{3} s, \ldots, r^{n-1} s\right\}$. Then $\left(A_{1}, A_{2}, A_{3}\right)$ is a complete decomposition of $D_{2 n}$ of order 3 .

\section{Proof.}

We first compute $A_{1} A_{2}$ as follows:

and followed by

$$
\begin{aligned}
A_{1} A_{2} & =\{1, r\}\{s, r s\} \\
& =\left\{s, r s, r^{2} s\right\}
\end{aligned}
$$

$$
\begin{aligned}
A_{1} A_{2} A_{3}= & \left\{s, r s, r^{2} s\right\}\left\{r^{2}, r^{3}, \ldots, r^{n-1}, r^{2} s, r^{3} s, \ldots, r^{n-1} s\right\} \\
= & \left\{r^{n-2} s, r^{n-3} s, \ldots, r s, r^{n-2}, r^{n-3}, \ldots, r\right. \\
& r^{n-1} s, r^{n-2} s, \ldots, r^{2} s, r^{n-1}, r^{n-2}, \ldots, r^{2} \\
& \left.r^{n} s, r^{n-1} s, \ldots, r^{3} s, r^{n}, r^{n-1}, \ldots, r^{3}\right\} \\
= & \left\{1, r, \ldots, r^{n-1}, s, r s, \ldots, r^{n-1} s\right\}=D_{2 n} .
\end{aligned}
$$

Hence, $\left(A_{1}, A_{2}, A_{3}\right)$ is a complete decomposition of $D_{2 n}$ of order 3 .

Now, we shall focus on the construction, where $\left(A_{1}, A_{2}, \ldots, A_{k}\right)$ is a complete decomposition of $D_{2 n}$ of order $k \in\{4,5, \ldots, n\}$. The constructions of complete decompositions of $D_{2 n}$ of order $k$ for $k \in\{4,5, \ldots, n\}$ are as follows:

$$
\begin{aligned}
& A_{1}=\{1, r\} ; \\
& A_{2}=\{s, r s\} \\
& A_{i}=\left\{r^{i-1}, r^{i-1} s\right\} \text { for } i \in\{3,4, \ldots, k-1\} ; \\
& A_{k}=\left\{r^{k-1}, r^{k}, \ldots, r^{n-1}, r^{k-1} s, r^{k} s, \ldots, r^{n-1} s\right\} .
\end{aligned}
$$


Proposition 6. Let $n \geq 7$ be integer. There exists a complete decomposition of $D_{2 n}$ of order $k$ for $k \in\{4,5,6,7\}$.

\section{Proof.}

Let

$$
\begin{aligned}
A_{1} & =\{1, r\} ; \\
A_{2} & =\{s, r s\} ; \\
A_{i} & =\left\{r^{i-1}, r^{i-1} s\right\}, \quad \text { for } i \in\{3,4, \ldots, k-1\} ; \\
A_{k} & =\left\{r^{k-1}, r^{k}, \ldots, r^{n-1}, r^{k-1} s, r^{k} s, \ldots, r^{n-1} s\right\} .
\end{aligned}
$$

where $A_{1}, A_{2}, \ldots, A_{k} \subseteq D_{2 n}$. We separate the proof into 4 cases for $k \in\{4,5,6,7\}$ respectively. Note that $A_{1} A_{2}=\{1, r\}\{s, r s\}=\left\{s, r s, r^{2} s\right\}$. We compute $A_{1} A_{2} A_{3}$ as follows:

$$
A_{1} A_{2} A_{3}=\left\{s, r s, r^{2} s\right\}\left\{r^{2}, r^{2} s\right\}=\left\{1, r^{n-2}, r^{n-1}, s, r^{n-2} s, r^{n-1} s\right\}
$$

(i) When $k=4$, we have $A_{4}=\left\{r^{3}, r^{4}, \ldots, r^{n-1}, r^{3} s, r^{4} s, \ldots, r^{n-1} s\right\}$. We compute $A_{1} A_{2} A_{3} A_{4}$ as follows:

$$
\begin{aligned}
A_{1} A_{2} A_{3} A_{4} & =\left\{1, r^{n-2}, r^{n-1}, s, r^{n-2} s, r^{n-1} s\right\}\left\{r^{3}, r^{4}, \ldots, r^{n-1}, r^{3} s, r^{4} s, \ldots, r^{n-1} s\right\} \\
& =\left\{1, r, \ldots, r^{n-1}, s, r s, \ldots, r^{n-1} s\right\}=D_{2 n} .
\end{aligned}
$$

Hence, $\left(A_{1}, A_{2}, A_{3}, A_{4}\right)$ is a complete decomposition of $D_{2 n}$ of order 4 .

(ii) When $k=5$, we have $A_{4}=\left\{r^{3}, r^{3} s\right\}$. From Equation (1), we have $A_{1} A_{2} A_{3}=$ $\left\{1, r^{n-2}, r^{n-1}, s, r^{n-2} s, r^{n-1} s\right\}$. Now, we compute $A_{1} A_{2} A_{3} A_{4}$ as follows:

$$
\begin{aligned}
& A_{1} A_{2} A_{3} A_{4} \\
& =\left\{1, r^{n-2}, r^{n-1}, s, r^{n-2} s, r^{n-1} s\right\}\left\{r^{3}, r^{3} s\right\} \\
& =\left\{r, r^{2}, r^{3}, r^{n-5}, r^{n-4}, r^{n-3}, r s, r^{2} s, r^{3} s, r^{n-5} s, r^{n-4} s, r^{n-3} s\right\}
\end{aligned}
$$

Observe that $A_{5}=\left\{r^{4}, r^{5}, \ldots, r^{n-1}, r^{4} s, r^{5} s, \ldots, r^{n-1} s\right\}$. We compute $A_{1} A_{2} A_{3} A_{4} A_{5}$ as follows:

$$
\begin{aligned}
& A_{1} A_{2} A_{3} A_{4} A_{5} \\
= & \left\{r, r^{2}, r^{3}, r^{n-5}, r^{n-4}, r^{n-3}, r s, r^{2} s, r^{3} s, r^{n-5} s, r^{n-4} s, r^{n-3} s\right\}\left\{r^{4}, r^{5},\right. \\
& \left.\ldots, r^{n-1}, r^{4} s, r^{5} s, \ldots, r^{n-1} s\right\} \\
= & \left\{1, r, \ldots, r^{n-1}, s, r s, \ldots, r^{n-1} s\right\}=D_{2 n} .
\end{aligned}
$$

Hence, $\left(A_{1}, A_{2}, A_{3}, A_{4}, A_{5}\right)$ is a complete decomposition of $D_{2 n}$ of order 5 .

(iii) When $k=6$, we have $A_{5}=\left\{r^{4}, r^{4} s\right\}$. From Equation (2), we have

$$
A_{1} A_{2} A_{3} A_{4}=\left\{r, r^{2}, r^{3}, r^{n-5}, r^{n-4}, r^{n-3}, r s, r^{2} s, r^{3} s, r^{n-5} s, r^{n-4} s, r^{n-3} s\right\}
$$

Then, we compute $A_{1} A_{2} A_{3} A_{4} A_{5}$ as follows:

$$
\begin{aligned}
& A_{1} A_{2} A_{3} A_{4} A_{5} \\
&=\left\{r, r^{2}, r^{3}, r^{n-5}, r^{n-4}, r^{n-3}, r s, r^{2} s, r^{3} s, r^{n-5} s, r^{n-4} s, r^{n-3} s\right\}\left\{r^{4}, r^{4} s\right\} \\
&=\left\{1, r, r^{5}, r^{6}, r^{7}, r^{n-9}, r^{n-8}, r^{n-7}, r^{n-3}, r^{n-2}, r^{n-1}\right. \\
&\left.s, r s, r^{5} s, r^{6} s, r^{7} s, r^{n-9} s, r^{n-8} s, r^{n-7} s, r^{n-3} s, r^{n-2} s, r^{n-1} s\right\}
\end{aligned}
$$

Note that $A_{6}=\left\{r^{5}, r^{6}, \ldots, r^{n-1}, r^{5} s, r^{6} s, \ldots, r^{n-1} s\right\}$. Next, we compute $A_{1} A_{2} \ldots A_{6}$ as follows: 


$$
\begin{aligned}
& A_{1} A_{2} A_{3} A_{4} A_{5} A_{6} \\
= & \left\{1, r, r^{5}, r^{6}, r^{7}, r^{n-9}, r^{n-8}, r^{n-7}, r^{n-3}, r^{n-2}, r^{n-1},\right. \\
& \left.s, r s, r^{5} s, r^{6} s, r^{7} s, r^{n-9} s, r^{n-8} s, r^{n-7} s, r^{n-3} s, r^{n-2} s, r^{n-1} s\right\} \\
& \left\{r^{5}, r^{6}, \ldots, r^{n-1}, r^{5} s, r^{6} s, \ldots, r^{n-1} s\right\} \\
= & \left\{1, r, \ldots, r^{n-1}, s, r s, \ldots, r^{n-1} s\right\}=D_{2 n} .
\end{aligned}
$$

Hence, the result holds.

(iv) When $k=7$, we have $A_{6}=\left\{r^{5}, r^{5} s\right\}$. By using the similar way, it can be shown that $\left(A_{1}, A_{2}, \ldots, A_{7}\right)$ is a complete decomposition of $D_{2 n}$ of order 7 .

The following lemma shall be used in later proof.

Lemma 7. Let $n \geq 8, k \in\{8,9, \ldots, n\}$ and $A_{i}=\left\{r^{i-1}, r^{i-1} s\right\} \subseteq D_{2 n}$ for $i \in\{3,4, \ldots, k-1\}$. Then $A_{3} A_{4} \ldots A_{k-1}=\left\{r^{-c+5}, r^{-c+11}, r^{-c+13}, \ldots, r^{c-9}, r^{c-7}, \ldots, r^{c-1}\right\} \cup\left\{r^{-c+5} s, r^{-c+11} s\right.$, $\left.r^{-c+13} s, \ldots, r^{c-9} s, r^{c-7} s, r^{c-1} s\right\}$, where $c=\frac{(k-2)(k-1)}{2}$.

\section{Proof.}

We first show that the base case $k=8$ is true. We compute $A_{3} A_{4} A_{5} A_{6} A_{7}$ as follows:

$$
\begin{aligned}
A_{3} A_{4}= & \left\{r^{2}, r^{2} s\right\}\left\{r^{3}, r^{3} s\right\}=\left\{r^{-1}, r^{5}, r^{-1} s, r^{5} s\right\} \\
A_{3} A_{4} A_{5}= & \left\{r^{-1}, r^{5}, r^{-1} s, r^{5} s\right\}\left\{r^{4}, r^{4} s\right\}=\left\{r^{-5}, r, r^{3}, r^{9}, r^{-5} s, r s, r^{3} s, r^{9} s\right\} \\
A_{3} A_{4} A_{5} A_{6}=\left\{r^{-5}, r, r^{3}, r^{9}, r^{-5} s, r s, r^{3} s, r^{9} s\right\}\left\{r^{5}, r^{5} s\right\} & =\left\{r^{-10}, r^{-4}, r^{-2}, 1, r^{4}, r^{6}, r^{8}, r^{14}, r^{-10} s, r^{-4} s, r^{-2} s, s, r^{4} s, r^{6} s, r^{8} s, r^{14} s\right\} \\
A_{3} A_{4} A_{5} A_{6} A_{7} & \\
= & \left\{r^{-10}, r^{-4}, r^{-2}, 1, r^{4}, r^{6}, r^{8}, r^{14}, r^{-10} s, r^{-4} s, r^{-2} s, s, r^{4} s, r^{6} s, r^{8} s, r^{14} s\right\}\left\{r^{6}, r^{6} s\right\} \\
= & \left\{r^{-16}, r^{-10}, r^{-8}, r^{-6}, r^{-4}, r^{-2}, 1, r^{2}, r^{4}, r^{6}, r^{8}, r^{10}, r^{12}, r^{14}, r^{20}\right\} \cup \\
& \left\{r^{-16} s, r^{-10} s, r^{-8} s, r^{-6} s, r^{-4} s, r^{-2} s, s, r^{2} s, r^{4} s, r^{6} s, r^{8} s, r^{10} s, r^{12} s, r^{14} s, r^{20} s\right\}
\end{aligned}
$$

Clearly the statement is true when $k=8$. Thus the base case holds. Now, we assume that the statement is true when $k=m$. By assumption, it is clear that

where

$$
\begin{aligned}
& A_{3} A_{4} \ldots A_{m-1} \\
= & \left\{r^{-\frac{(m-2)(m-1)}{2}+5}, r^{-\frac{(m-2)(m-1)}{2}+11}, r^{-\frac{(m-2)(m-1)}{2}+13}, \ldots,\right. \\
& \left.r^{\frac{(m-2)(m-1)}{2}-9}, r^{\frac{(m-2)(m-1)}{2}-7}, r^{\frac{(m-2)(m-1)}{2}-1}\right\} \cup \\
& \left\{r^{-\frac{(m-2)(m-1)}{2}+5} s, r^{-\frac{(m-2)(m-1)}{2}+11} s, r^{-\frac{(m-2)(m-1)}{2}+13} s, \ldots,\right. \\
& \left.r^{\frac{(m-2)(m-1)}{2}-9} s, r^{\frac{(m-2)(m-1)}{2}-7} s, r^{\frac{(m-2)(m-1)}{2}-1} s\right\} \\
= & B_{1} \cup B_{1} s,
\end{aligned}
$$

$$
\begin{gathered}
B_{1}=\left\{r^{-\frac{(m-2)(m-1)}{2}+5}, r^{-\frac{(m-2)(m-1)}{2}+11}, r^{-\frac{(m-2)(m-1)}{2}+13}, \ldots,\right. \\
\left.r^{\frac{(m-2)(m-1)}{2}-9}, r^{\frac{(m-2)(m-1)}{2}-7}, r^{\frac{(m-2)(m-1)}{2}-1}\right\} .
\end{gathered}
$$

Next, we show that the statement is also true for $k=m+1$. Note that $A_{m}=$ $\left\{r^{m-1}, r^{m-1} s\right\}$ and $A_{3} A_{4} \ldots A_{m}=B_{1} A_{m} \cup B_{1} s A_{m}$. We compute $B_{1} A_{m}$ and $B_{1} s A_{m}$ as follows: 
where

$$
\begin{aligned}
B_{1} A_{m}= & \left\{r^{-\frac{(m-2)(m-1)}{2}+5}, r^{-\frac{(m-2)(m-1)}{2}+11}, r^{-\frac{(m-2)(m-1)}{2}+13}, \ldots,\right. \\
& \left.r^{\frac{(m-2)(m-1)}{2}-9}, r^{\frac{(m-2)(m-1)}{2}-7}, r^{\frac{(m-2)(m-1)}{2}-1}\right\}\left\{r^{m-1}, r^{m-1} s\right\} \\
= & \left\{r^{-\frac{(m-2)(m-1)}{2}+5+(m-1)}, r^{-\frac{(m-2)(m-1)}{2}+11+(m-1)}, r^{-\frac{(m-2)(m-1)}{2}+13+(m-1)}, \ldots,\right. \\
= & C_{1} \cup C_{1} s \\
B_{1} S A_{m}= & \left\{r^{-\frac{(m-2)(m-1)}{2}+5} s, r^{-\frac{(m-2)(m-1)}{2}+11} s, r^{-\frac{(m-2)(m-1)}{2}+13} s, \ldots,\right. \\
& \left.r^{\frac{(m-2)(m-1)}{2}-9} s, r^{\frac{(m-2)(m-1)}{2}-7} s, r^{\frac{(m-2)(m-1)}{2}-1} s\right\}\left\{r^{m-1}, r^{m-1} s\right\} \\
= & \left\{r^{-\frac{(m-2)(m-1)}{2}+5-(m-1)}, r^{-\frac{(m-2)(m-1)}{2}+11-(m-1)}, r^{-\frac{(m-2)(m-1)}{2}+13-(m-1)}, \ldots,\right. \\
& \left.r^{\frac{(m-2)(m-1)}{2}-9-(m-1)}, r^{\frac{(m-2)(m-1)}{2}-7-(m-1)}, r^{\frac{(m-2)(m-1)}{2}-1-(m-1)}\right\}\{1, s\} \\
= & D_{1} \cup D_{1} s,
\end{aligned}
$$

$$
\begin{aligned}
& C_{1} \\
& =\left\{r^{-\frac{(m-2)(m-1)}{2}+5+(m-1)}, r^{-\frac{(m-2)(m-1)}{2}+11+(m-1)}, r^{-\frac{(m-2)(m-1)}{2}+13+(m-1)}, \ldots,\right. \\
& \left.r^{\frac{(m-2)(m-1)}{2}-9+(m-1)}, r^{\frac{(m-2)(m-1)}{2}-7+(m-1)}, r^{\frac{(m-2)(m-1)}{2}-1+(m-1)}\right\} \\
& =\left\{r^{-\frac{m(m-1)}{2}+2 m+3}, r^{-\frac{m(m-1)}{2}+2 m+9}, \ldots, r^{-\frac{m(m-1)}{2}+2 m+11}, \ldots, r^{\frac{m(m-1)}{2}-9}, r^{\frac{m(m-1)}{2}-7}, \ldots, r^{\frac{m(m-1)}{2}-1}\right\} \text {, } \\
& D_{1}=\left\{r^{-\frac{(m-2)(m-1)}{2}+5-(m-1)}, r^{-\frac{(m-2)(m-1)}{2}+11-(m-1)}, r^{-\frac{(m-2)(m-1)}{2}+13-(m-1)}, \ldots,\right. \\
& \left.r^{\frac{(m-2)(m-1)}{2}-9-(m-1)}, r^{\frac{(m-2)(m-1)}{2}-7-(m-1)}, r^{\frac{(m-2)(m-1)}{2}-1-(m-1)}\right\} \\
& =\left\{r^{-\frac{m(m-1)}{2}+5}, r^{-\frac{m(m-1)}{2}+11}, r^{-\frac{m(m-1)}{2}+13}, \ldots, r^{\frac{m(m-1)}{2}-9}, r^{\frac{m(m-1)}{2}-7}, r^{\frac{m(m-1)}{2}-1}\right\} .
\end{aligned}
$$

Thus, we have

$C_{1} \cup D_{1}=\left\{r^{-\frac{m(m-1)}{2}+5}, r^{-\frac{m(m-1)}{2}+11}, r^{-\frac{m(m-1)}{2}+13}, \ldots r^{\frac{m(m-1)}{2}-9}, r^{\frac{m(m-1)}{2}-7}, r^{\frac{m(m-1)}{2}-1}\right\}$, $C_{1} s \cup D_{1} s$

$=\left\{r^{-\frac{m(m-1)}{2}+5} s, r^{-\frac{m(m-1)}{2}+11} s, r^{-\frac{m(m-1)}{2}+13} s, \ldots, r^{\frac{m(m-1)}{2}-9} s, r^{\frac{m(m-1)}{2}-7} s, r^{\frac{m(m-1)}{2}-1} s\right\}$.

Note that $A_{3} A_{4} \ldots A_{m}=C_{1} \cup D_{1} \cup C_{1} s \cup D_{1} s$. Therefore, the statement is also true for $k=$ $m+1$. Hence, by mathematical induction, the statement is true for $k \in\{8,9, \ldots, n\}$.

Theorem 8. Let $n \geq 8$. There exists a complete decomposition of $D_{2 n}$ of order $k$ for $k \in$ $\{8,9, \ldots, n\}$.

\section{Proof.}

Let $A_{1}=\{1, r\}, A_{2}=\{s, r s\}, A_{i}=\left\{r^{i-1}, r^{i-1} s\right\}$ for $i \in\{3,4, \ldots, k-1\}$ and $A_{k}=$ $\left\{r^{k-1}, r^{k}, \ldots, r^{n-1}, r^{k-1} s, r^{k} s, \ldots, r^{n-1} s\right\}$ be the subsets of $D_{2 n}$. We first compute

Let $c=\frac{(k-2)(k-1)}{2}$. By Lemma 7, we have

$$
A_{1} A_{2}=\{1, r\}\{s, r s\}=\left\{s, r s, r^{2} s\right\}
$$

$$
\begin{aligned}
A_{3} A_{4} \ldots A_{k-1}= & \left\{r^{-c+5}, r^{-c+11}, r^{-c+13}, \ldots, r^{c-9}, r^{c-7}, r^{c-1}\right\} \cup \\
& \left\{r^{-c+5} s, r^{-c+11} s, r^{-c+13} s, \ldots, r^{c-9} s, r^{c-7} s, r^{c-1} s\right\} \\
= & L_{1} \cup L_{2},
\end{aligned}
$$

where

$$
L_{1}=\left\{r^{-c+5}, r^{-c+11}, r^{-c+13}, \ldots, r^{c-9}, r^{c-7}, r^{c-1}\right\}
$$

and

$$
L_{2}=\left\{r^{-c+5} s, r^{-c+11} s, r^{-c+13} s, \ldots, r^{c-9} s, r^{c-7} s, r^{c-1} s\right\} \text {. }
$$

Now, we compute $A_{1} A_{2} L_{1} \subseteq A_{1} A_{2} \ldots A_{k-1}$ and $A_{1} A_{2} L_{2} \subseteq A_{1} A_{2} \ldots A_{k-1}$ as follows: 
where

$$
\begin{aligned}
A_{1} A_{2} L_{1}= & \left\{s, r s, r^{2} s\right\}\left\{r^{-c+5}, r^{-c+11}, r^{-c+13}, \ldots, r^{c-9}, r^{c-7}, r^{c-1}\right\} \\
= & \left\{r^{n-c+1} s, r^{n-c+2} s, r^{n-c+3} s\right\} \cup\left\{r^{n-c+7} s, r^{n-c+8} s, \ldots, r^{n+c-9} s\right\} \cup \\
& \left\{r^{n+c-5} s, r^{n+c-4} s, r^{n+c-3} s\right\} \\
= & P_{1} \cup P_{2} \cup P_{3} \\
A_{1} A_{2} L_{2}= & \left\{s, r s, r^{2} s\right\}\left\{r^{-c+5} s, r^{-c+11} s, r^{-c+13} s, \ldots, r^{c-9} s, r^{c-7} s, r^{c-1} s\right\} \\
= & \left\{r^{n-c+1}, r^{n-c+2}, r^{n-c+3}\right\} \cup\left\{r^{n-c+7}, r^{n-c+8}, \ldots, r^{n+c-9}\right\} \cup \\
& \left\{r^{n+c-5}, r^{n+c-4}, r^{n+c-3}\right\} \\
= & M_{1} \cup M_{2} \cup M_{3},
\end{aligned}
$$

$$
\begin{aligned}
P_{1} & =\left\{r^{n-c+1} s, r^{n-c+2} s, r^{n-c+3} s\right\}, \\
P_{2} & =\left\{r^{n-c+7} s, r^{n-c+8} s, \ldots, r^{n+c-9} s\right\}, \\
P_{3} & =\left\{r^{n+c-5} s, r^{n+c-4} s, r^{n+c-3} s\right\}, \\
M_{1} & =\left\{r^{n-c+1}, r^{n-c+2}, r^{n-c+3}\right\}, \\
M_{2} & =\left\{r^{n-c+7}, r^{n-c+8}, \ldots, r^{n+c-9}\right\}, \\
M_{3} & =\left\{r^{n+c-5}, r^{n+c-4}, r^{n+c-3}\right\} .
\end{aligned}
$$

Now, we compute $P_{1} A_{k}, P_{2} A_{k}, \ldots, P_{6} A_{k} \subseteq A_{1} A_{2} \ldots A_{k}$ as follows:

$$
\begin{aligned}
& P_{1} A_{k} \\
& =\left\{r^{n-c+1} s, r^{n-c+2} s, r^{n-c+3} s\right\}\left\{r^{k-1}, r^{k}, \ldots, r^{n-1}, r^{k-1} s, r^{k} s, \ldots, r^{n-1} s\right\} \\
& =\left\{r^{n-c+2}, r^{n-c+3}, \ldots, r^{2 n-c-k+4}\right\} \cup\left\{r^{n-c+2} s, r^{n-c+3} s, \ldots, r^{2 n-c-k+4} s\right\} \\
& =R_{1} \text {; } \\
& P_{2} A_{k} \\
& =\left\{r^{n-c+7} s, r^{n-c+8} s, r^{n+c+9} s\right\}\left\{r^{k-1}, r^{k}, \ldots, r^{n-1}, r^{k-1} s, r^{k} s, \ldots, r^{n-1} s\right\} \\
& =\left\{r^{n-c+8}, r^{n-c+9}, \ldots, r^{2 n+c-k-8}\right\} \cup\left\{r^{n-c+8} s, r^{n-c+9} s, \ldots, r^{2 n+c-k-8} s\right\} \\
& =R_{2} \text {; } \\
& P_{3} A_{k} \\
& =\left\{r^{n+c-5} s, r^{n+c-4} s, r^{n+c-3} s\right\}\left\{r^{k-1}, r^{k}, \ldots, r^{n-1}, r^{k-1} s, r^{k} s, \ldots, r^{n-1} s\right\} \\
& =\left\{r^{n+c-4}, r^{n+c-3}, \ldots, r^{2 n+c-k-2}\right\} \cup\left\{r^{n+c-4} s, r^{n+c-3} s, \ldots, r^{2 n+c-k-2} s\right\} \\
& =R_{3} \text {; } \\
& M_{1} A_{k} \\
& =\left\{r^{n-c+1}, r^{n-c+2}, r^{n-c+3}\right\}\left\{r^{k-1}, r^{k}, \ldots, r^{n-1}, r^{k-1} s, r^{k} s, \ldots, r^{n-1} s\right\} \\
& =\left\{r^{n-c+k}, r^{n-c+k+1}, \ldots, r^{2 n-c+2}\right\} \cup\left\{r^{n-c+k} s, r^{n-c+k+1} s, \ldots, r^{2 n-c+2} s\right\} \\
& =R_{4} \text {; } \\
& M_{2} A_{k} \\
& =\left\{r^{n-c+7}, r^{n-c+8}, \ldots, r^{n+c-9}\right\}\left\{r^{k-1}, r^{k}, \ldots, r^{n-1}, r^{k-1} s, r^{k} s, \ldots, r^{n-1} s\right\} \\
& =\left\{r^{n-c+k+6}, r^{n-c+k+7}, \ldots, r^{2 n+c-10} \cup\left\{r^{n-c+k+6} s, r^{n-c+k+7} s, \ldots, r^{2 n+c-10} s\right\}\right. \\
& =R_{5} \text {; } \\
& M_{3} A_{k} \\
& =\left\{r^{n+c-5}, r^{n+c-4}, r^{n+c-3}\right\}\left\{r^{k-1}, r^{k}, \ldots, r^{n-1}, r^{k-1} s, r^{k} s, \ldots, r^{n-1} s\right\} \\
& =\left\{r^{n+c-k-6}, r^{n+c-k-5}, \ldots, r^{2 n+c-4}\right\} \cup\left\{r^{n+c-k-6} s, r^{n+c-k-5} s, \ldots, r^{2 n+c-4} s\right\} \\
& =R_{6} \text {. }
\end{aligned}
$$

Note that $A_{1} A_{2} \ldots A_{k}=R_{1} \cup R_{2} \cup R_{3} \cup R_{4} \cup R_{5} \cup R_{6}$. Observe that

$$
R_{2}=\left\{r^{n-c+8}, r^{n-c+9}, \ldots, r^{2 n+c-k-8}\right\} \cup\left\{r^{n-c+8} s, r^{n-c+9} s, \ldots, r^{2 n+c-k-8} s\right\},
$$


where

$$
\begin{aligned}
\left|\left\{r^{n-c+8}, r^{n-c+9}, \ldots, r^{2 n+c-k-8}\right\}\right| & =\left|\left\{r^{n-c+8} s, r^{n-c+9} s, \ldots, r^{2 n+c-k-8} s\right\}\right| \\
& =n+2 c-k-15 \\
& =n+k^{2}-2 k-15 \geq n
\end{aligned}
$$

for $k \in\{8,9, \ldots, n\}$. Therefore, we have $R_{2}=Q_{2}$. Hence, there exists a complete decomposition of $D_{2 n}$ of order $k$ for $k \in\{8,9, \ldots, n\}$.

The research has been carried out under Fundamental Research Grant Scheme project FRGS/1/2017/STG06/UTAR/02/3 provided by Ministry of Higher Education of Malaysia.

\section{References}

1. A.L. Cauchy, J. Ecole Polytechniques 9, 1 - 11 (1813)

2. H. Davenport, J. London Math. Soc. 10, 30 - 32 (1935)

3. G. Hajos, Mat. Fiz. Lapok 45, $171-190$ (1938)

4. G. Hajos, Math. Zeit 47, 427 - 467 (1942)

5. H. Minkowski, Geometrie der Zahlen, Teubner, Leipzig (1896)

6. N.G. Bruijn, Indag. Math. 15, 370 - 377 (1953)

7. L. Redei, Acta Math. Acad. Sci. Hungar 16, 329 - 373 (1965)

8. T. Sharmini, Some properties of subsets of finite groups, Master Thesis, Universiti Tunku Abdul Rahman, Malaysia (2012)

9. D.C.K. Wong, K.W. Wong, W.S. Yap, Asian Euro. J. Math. 11(3), 1 - 13 (2018)

10. A.Y.M. Chin, H.V. Chen, Appl. Algebra Eng. Commun. Comput. 30(3), 263 - 274 (2018) 